\title{
Fluid Management in the Intensive Care Unit: Bioelectrical Impedance Vector Analysis as a Tool to Assess Hydration Status and Optimal Fluid Balance in Critically III Patients
}

\author{
Flavio Basso ${ }^{a}$ Giovanna Berdin ${ }^{a}$ Grazia Maria Virzì ${ }^{a, c}$ Giacomo Mason ${ }^{a}$ \\ Pasquale Piccinni $^{b}$ Sonya Day ${ }^{a}$ Dinna N. Cruz ${ }^{a}$ Marzena Wjewodzka ${ }^{a}$ \\ Anna Giuliani ${ }^{a} \quad$ Alessandra Brendolan ${ }^{a, c}$ Claudio Ronco ${ }^{a, c}$

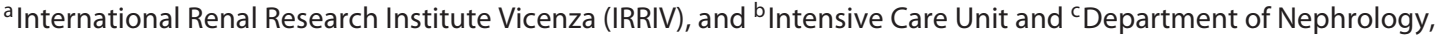 \\ San Bortolo Hospital, Vicenza, Italy
}

\section{Key Words}

Bioelectric impedance vector analysis · Continuous renal replacement therapy - Fluid overload · Hydration status . Intensive care

\begin{abstract}
Background: Fluid balance disorders are a relevant risk factor for morbidity and mortality in critically ill patients. Volume assessment in the intensive care unit (ICU) is thus of great importance, but there are currently few methods to obtain an accurate and timely assessment of hydration status. Our aim was to evaluate the hydration status of ICU patients via bioelectric impedance vector analysis (BIVA) and to investigate the relationship between hydration and mortality. Methods: We evaluated 280 BIVA measurements of 64 patients performed daily in the 5 days following their ICU admission. The observation period ranged from a minimum of $72 \mathrm{~h}$ up to a maximum of $120 \mathrm{~h}$. We observed the evolution of the hydration status during the ICU stay in this population, and analyzed the relationship between mean and maximum hydration reached and mortality - both in the ICU and at 60 days - using logistic regression. Results: A state of overhydration was observed in the majority of patients (70\%) on admission, which persisted during the ICU stay. Pa-
\end{abstract}

tients who required continuous renal replacement therapy (CRRT) were more likely to be overhydrated starting from the 2nd day of observation. Logistic regression showed a strong and significant correlation between mean/maximum hydration reached and mortality, both independently and correcting for severity of prognosis. Conclusions: Fluid overload measured by BIVA is a frequent condition in critically ill patients - whether or not they undergo CRRT - and a significant predictor of mortality. Hence, hydration status should be considered as an additional prognosticator in the clinical management of the critically ill patient. Key Messages: (i) On the day of ICU admittance, patients showed a marked tendency to overhydration ( $>70 \%$ of total). This tendency was more pronounced in patients on CRRT. (ii) Hyperhydration persisted during the ICU stay. Patients who underwent CRRT showed significantly higher hyperhydration from the 2nd day of hospitalization. (iii) Nonsurvivors showed worse hyperhydration patterns in comparison to survivors in logistic univariate analysis $(p<0.05)$. This relationship between hydration and mortality is confirmed even when controlling for the effect of a worse prognosis approximated by any of three ICU scoring systems (APACHE II, SAPS II and SOFA). Mean and maximum hydration levels present a stronger correlation with mortality than with mean and maximum cumulative fluid balance reached during the observation period.

(c) 2013 S. Karger AG, Base

Claudio Ronco, MD

Department of Nephrology, Dialysis and Transplantation

San Bortolo Hospital and International Renal Research Institute Vicenza (IRRIV)

Viale Rodolfi 37, IT-36100 Vicenza (Italy)

E-Mail cronco@goldnet.it 


\section{Introduction}

Hydration homeostasis in the intensive care setting is complex and requires in-depth knowledge of fluid balance dynamics. Deviation from normal values is associated with increased morbidity and mortality. Several multicenter clinical trials have shown a positive correlation between fluid overload and adverse outcomes in critically ill patients admitted to the intensive care unit (ICU) [1-4]. In the PICARD (Program to Improve Care in Acute Renal Disease) study, fluid overload is associated with a reduced likelihood of recovery of renal function in patients with acute kidney injury (AKI). Furthermore, mortality is much higher in AKI patients with excessive liquid accumulation [2]. It has also been shown that setting hydration goals in the ICU patient can improve outcome. Goal-directed therapies can decrease hospitalization and reduce complications [1]. A recent study has underlined that restrictive rather than liberal fluid therapy can improve patient outcome [1].

For these reasons, volume assessment is a fundamental element in the correct management of the critically ill patient. The limited availability of scale beds in the ICU often makes it difficult to monitor exact weight. At present, clinical tools used commonly for the determination and quantification of volume status in the ICU - each with its own limitations - are: (i) registration of fluid intake and output; (ii) clinical signs of fluid overload (body weight, peripheral edema and gas exchange parameters) and hemodynamic parameters of hypovolemia (hypotension, tachycardia, poor capillary refill and altered mental status) [5]; (iii) filling pressure (central venous pressure), and (iv) radiological techniques. Low accuracy has been demonstrated between the exact determination of body weight and the recording of fluid balance by the ICU staff [6]. Clinical parameters have been found to be poor and late indicators of overt volemia alterations $[7,8]$. As far as central venous pressure is concerned, it must be taken into account that the adequate circulating volume may depend on the size of the intravascular space and cardiac function [5]. The use of radiological imaging, in particular chest X-ray, is limited due to radiation exposure, and the evaluation has a large interobserver variation [9]. Therefore, other tools are required, which are able to give a reliable real-time estimate of the global hydration state.

Bioelectrical impedance analysis has been used for decades to measure resistivity of tissues, as well as to determine extracellular fluid volume and total body water. Bioimpedance is a measure of the body's conductivity and is directly proportional to its fluid composition [10]. Bio- electric impedance vector analysis (BIVA) combines measures of capacity with the standard bioimpedance. Total body impedance can be considered a combination of resistance $R$ (the opposition to the flow of an alternating current through intra- and extracellular electrolyte solutions) and reactance $X$ (the capacity produced by the interfaces of tissues and cell membranes) [11]. The two measurements, standardized for height $H$, can be displayed graphically: this generates an output that simultaneously reflects changes in hydration and soft tissue mass. This technology has been validated in depth in patients suffering from kidney disease: considering the three biggest studies on the subject, a total of $>4,000$ patients were analyzed [12-14].

BIVA is already implemented for the determination and monitoring of weight in the chronic kidney patient in both extracorporeal and peritoneal dialysis $[13,15,16]$. Nevertheless, there is currently limited evidence of the effectiveness of such instruments in the ICU setting. Some investigators have found a significant correlation between impedance measurements and clinical signs of hydration [17], whereas other authors have found that the bioimpedance method is not accurate enough to be the sole guide to fluid therapy [18]. However, the prognostic value of the fluid overload measured by BIVA in critically ill patients has not been investigated yet.

The aim of this study was to assess the effect of the BIVA-measured hydration status on mortality in critically ill patients. In particular, we wanted to investigate: (i) the hydration status of patients admitted to the ICU, and how this measure varies during hospitalization following current fluid management practices; (ii) the hydration status of ICU patients in need of renal replacement therapy (RRT), and how this varies during treatment, and (iii) the relationship between hydration and mortality with or without RRT.

\section{Materials and Methods}

\section{Study Design}

The study is a prospective assessment of the hydration state of adult patients in the ICU. This observational study comprised 64 patients observed from the first $24 \mathrm{~h}$ of ICU admission. The observation period ranged from a minimum of $72 \mathrm{~h}$ up to a maximum of $120 \mathrm{~h}$. Two hundred and eighty BIVA measurements were taken in the 64 patients. The BIVA hydration measurements were performed according to the manufacturer's instructions and taken by different trained operators in a blinded fashion each of the 5 days being observed. Research was conducted with the approval of the Institutional Review Board of the San Bortolo Hospital, Vicenza, Italy. We only included adult patients ( $\geq 18$ years) admitted 
to the ICU. The following exclusion criteria were applied: gravidity, presence of a defibrillator implant, extracorporeal membrane oxygenation, presence of bone fixation implants and limb amputations. Any patient not expected to survive $72 \mathrm{~h}$ or not expected to require at least $72 \mathrm{~h}$ of ICU hospitalization - as judged by the practicing clinician - was excluded.

Data Collection and Management

A database was used to store data for each patient, including anthropometric data, admission diagnosis and daily parameters such as systolic and diastolic blood pressure, mean arterial pressure, heart rate, urine output and fluid balance. AKI was diagnosed if the RIFLE criteria were met [19]. Body fluid status was assessed using standardized techniques with an electrical impedance analyzer. Data were graphically displayed using Cardio EFG software (Akern, Pontassieve, Italy). Patient measurements were collected using BIVA parameters according to the classical scheme of resistance, reactance and phase angle, as well as a percentage of body mass. The parameters were measured by an alternating electric current flux of $800 \mu \mathrm{A}$ and an operating frequency of $50 \mathrm{kHz}$.

Patient body fluid status was classified into three main categories: normally hydrated, dehydrated and hyperhydrated; the last two categories are further subdivided into mild, moderate or severe. The construction of a numerical scale arose from the need for a numerical value, which could be easily read and interpreted. In this scale, the normal level of hydration is set between 72.7 and 73.3\%. Higher and lower values represent states of hyperhydration and dehydration, respectively. Dehydration is classified into mild $(71-72.7 \%)$, moderate $(69-71 \%)$ and severe $(<69 \%)$. Similarly, overhydration is classified into mild $(74.4-81 \%)$, moderate (81$87 \%)$ and severe (>87\%) [20].

\section{Statistical Analysis}

Continuous variables were tested for normality via the ShapiroWilk test. Normally distributed variables were given as means \pm $\mathrm{SD}$, while those characterized by a non-normal distribution were given as medians (interquartile ranges). To analyze parameters such as the hydration status across groups (e.g. survivors and nonsurvivors), box plots were constructed representing the distribution of the variable in the two groups. Differences in continuous variables between groups were examined using Student's t test and Mann-Whitney's U test, depending on whether the variable in question was normally or nonnormally distributed. The Wilcoxon signed-rank test was used to test trends over time of hydration status. A value of $\mathrm{p}<0.05$ was considered statistically significant. The relationship between hydration status and mortality was finally analyzed using univariate and multivariate logistic regression.

\section{Results}

\section{Study Population}

Demographic characteristics, and clinical and laboratory values of the patients at the time of admission are summarized in table 1. On admission, there were no significant differences between patients grouped according to the hydration status, except for diastolic blood pressure and SAPS (Simplified Acute Physiology Score) II. The most frequent criteria for admission to the ICU during the study were sepsis (32.8\%) and heart failure (42.6\%). AKI occurred in $34.4 \%$ of the sample. Fourteen subjects (20.3\%) were started on continuous RRT (CRRT) during the ICU stay, of which 11 were started immediately on admission. The most common indication for CRRT was fluid overload with oliguria (11 of $14,78.6 \%)$ followed by sepsis (9 of $14,64.3 \%$ ). ICU mortality was $25 \%$. As expected, mortality was much higher in the 14 patients who required CRRT $(42.9 \%)$ compared to the patients who did not require RRT (18\%). The overall 60-day mortality was $31.3 \%, 57 \%$ for patients requiring CRRT and $24 \%$ for those who did not undergo the treatment.

\section{Hydration Trend during ICU Hospitalization}

More than $70 \%$ of patients were admitted to the ICU in a state of hyperhydration according to BIVA. The percentage of overhydrated patients remained around 70\% during the entire observation period (fig. 1). The mean hydration status is higher than the norm also when patients are stratified by CRRT. Moreover, patients who underwent CRRT showed significant increases in hydration in the ICU $(\mathrm{p}<0.05)$ compared to admission. While no statistically significant difference in hydration was seen between CRRT and non-CRRT patients on admission, the former present a higher hydration status in the remaining 4 days of observation ( $p<0.005$; fig. 2 ).

The mean and maximum hydration values achieved during monitoring were higher in patients who did not survive in the ICU (fig. 3). Similar to the CRRT and nonCRRT patients, mean hydration status was not different on admission, but a statistically significant difference $(\mathrm{p}<0.05)$ was noted in the following 3 days (fig. 4$)$.

\section{Outcome}

Univariate logistic regression shows a significant correlation between ICU mortality and maximum hydration (table 2): each added percentage point of maximum hydration status is associated with a higher probability of death of $2.64 \%$ (CI $0.62-4.65, \mathrm{p}<0.01$ ). A similar correlation is found between ICU mortality and mean hydration, where a single point increase in mean hydration corresponds to a $2.90 \%$ increase in the likelihood of death (CI $0.72-5.08, \mathrm{p}<0.01)$. The correlation between death and hydration remains significant even in a bivariate perspective, with the severity of the patients' prognosis included in the regression analysis. The coefficient of hydration remains significant regardless of which of the 3 ICU scoring systems [APACHE (Acute Physiology and Chronic Health Evaluation) II, SAPS II or SOFA (Sequential Organ Failure Assessment)] is used to stratify the analysis. 
Table 1. Clinical characteristics of the patients studied on admission

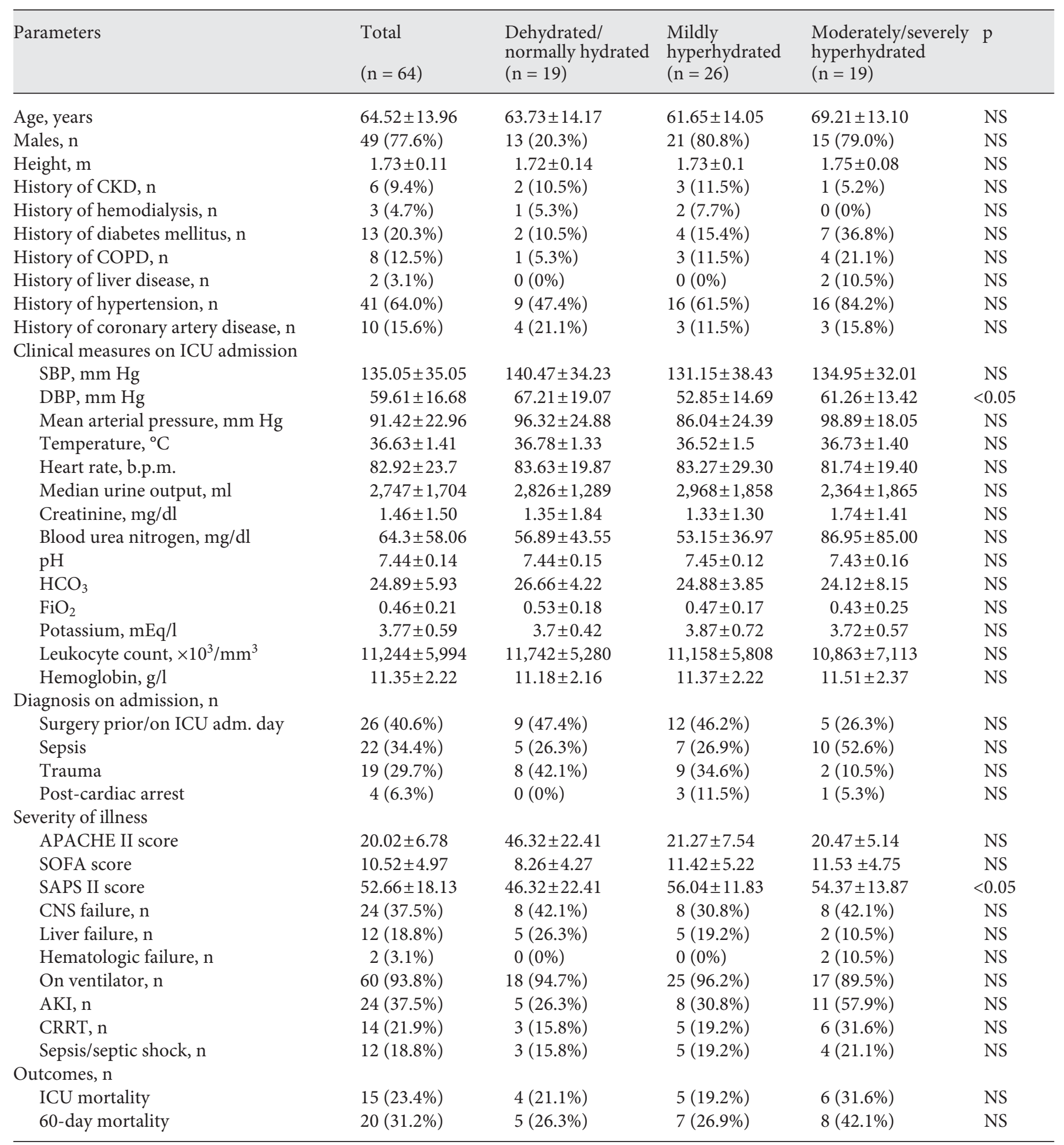

$\mathrm{COPD}=$ Chronic obstructive pulmonary disease; $\mathrm{SBP}=$ systolic blood pressure; $\mathrm{DBP}=$ diastolic blood pressure. 
Table 2. Univariate and bivariate logistic regression

\begin{tabular}{lllll}
\hline Outcome & Univariate & Bivariate & & \\
\cline { 3 - 5 } & & APACHE II & SAPS II & SOFA \\
\hline ICU mortality & & & & \\
$\quad$ Max. Hyd. in 5 days & $1.17^{*}(1.03-1.34)$ & $1.16^{*}(1.00-1.35)$ & $1.16^{*}(1.01-1.33)$ & $1.16^{*}(1.02-1.33)$ \\
Mean Hyd. in 5 days & $1.19^{*}(1.04-1.37)$ & $1.18^{*}(1.01-1.38)$ & $1.18^{*}(1.02-1.36)$ & $1.18^{*}(1.03-1.36)$ \\
Max. CFB in 5 days & $1.22(0.98-1.52)$ & $1.15(0.90-1.47)$ & $1.11(0.87-1.42)$ & $1.18(0.94-1.49)$ \\
Mean CFB in 5 days & $1.18(0.91-1.54)$ & $1.08(0.81-1.45)$ & $1.06(0.80-1.41)$ & $1.13(0.86-1.49)$ \\
60-day mortality & & & & \\
Max. Hyd. in 5 days & $1.16^{*}(1.03-1.30)$ & $1.14^{*}(1.00-1.29)$ & $1.14^{*}(1.01-1.29)$ & $1.15^{*}(1.02-1.30)$ \\
$\quad \begin{array}{l}\text { Mean Hyd. in 5 days } \\
\text { Max. CFB in 5 days }\end{array}$ & $1.17^{*}(1.03-1.33)$ & $1.15^{*}(1.01-1.32)$ & $1.16^{*}(1.02-1.32)$ & $1.17^{*}(1.03-1.34)$ \\
Mean CFB in 5 days & $1.14(0.93-1.39)$ & $1.07(0.86-1.33)$ & $1.06(0.85-1.33)$ & $1.13(0.91-1.40)$ \\
\hline
\end{tabular}

Odds ratios and 95\% confidence intervals of maximal and mean hydration (Hyd., in \%) and cumulative fluid balance (CFB, in liters) are reported. ${ }^{*} \mathrm{p}<0.05$.

The same type of regression using cumulative fluid balance in place of hydration levels shows a weaker correlation: to each additional liter of cumulative fluid balance corresponds a higher probability of death, although the difference was not significant $(\mathrm{p}=0.08)$. The result does not change even after adjustment for the severity of the prognosis.

We performed the same logistic analysis using 60-day mortality as an outcome instead of ICU mortality. Again, hydration during ICU was a significant predictor of mortality for both the maximum and the mean value (table 2 ).

\section{Discussion}

Total body fluid is inversely proportional to conductivity, a bioelectrical property of the body. The principle of BIVA is the quantification of the electrical conductivity of the body by measuring the resistance and reactance and correcting for height. Resistance and reactance are represented in a special graph known as a normogram: the shorter the resulting vector, the higher the content of body fluids. BIVA was originally applied to healthy individuals in order to establish normal values for resistance and reactance. These values have been used to construct confidence intervals in the form of ellipses $[21,22]$. A special software was developed to finally convert these parameters into a synthetic measure of body hydration percentage, which allows for a simpler interpretation.

The relationship between positive fluid balance and adverse outcomes in critically ill patients has already been

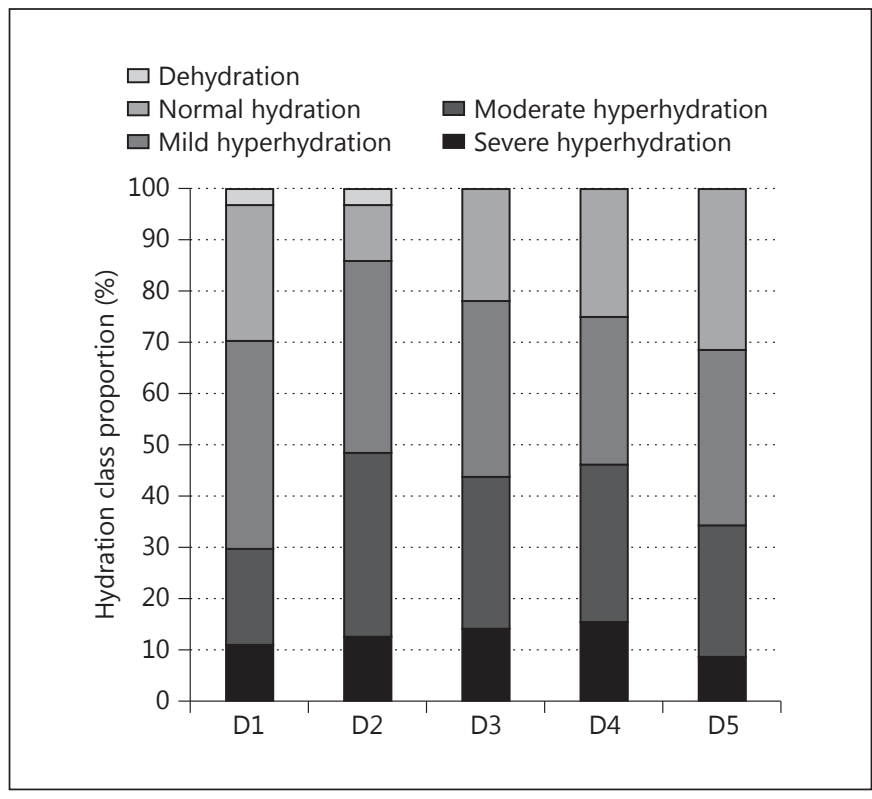

Fig. 1. Distribution of the hydration status in the study patients during the first 5 days in the ICU (D1-D5).

described in several studies [1, 23-27]. It was also demonstrated that a protocol restriction of infusions decreases the need for ventilation and length of ICU admission [3, $28,29]$. In a large, multicenter, observational study, nonsurvivors with acute lung injury had a higher fluid balance during the first 4 days as well as a longer mean ICU stay and cumulative fluid balance compared with survivors [29]. In a randomized study, Wiedemann et al. [3] com- 


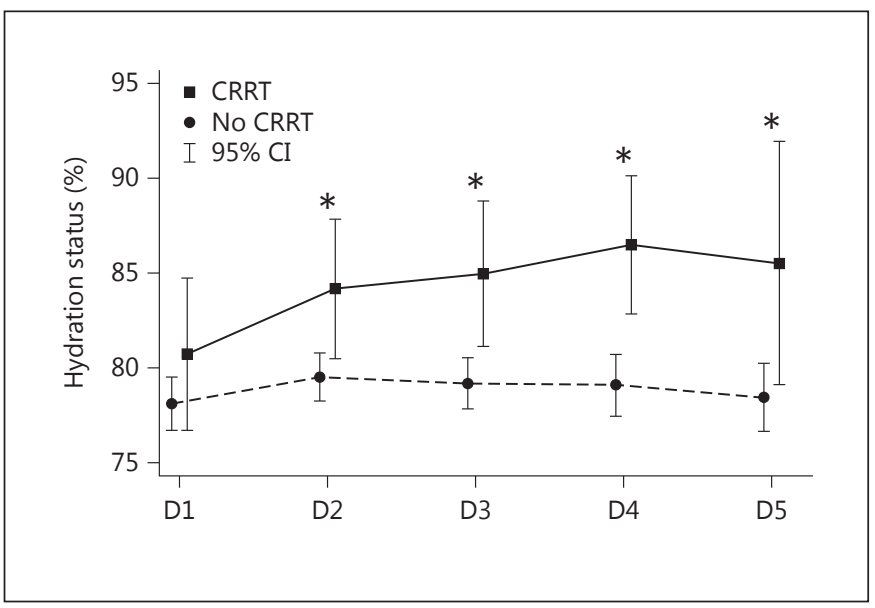

Fig. 2. Mean hydration status during the 5 -day observation period (D1-D5) for patients with/without CRRT ( ${ }^{*} \mathrm{p}<0.05$, Mann-Whitney U test).

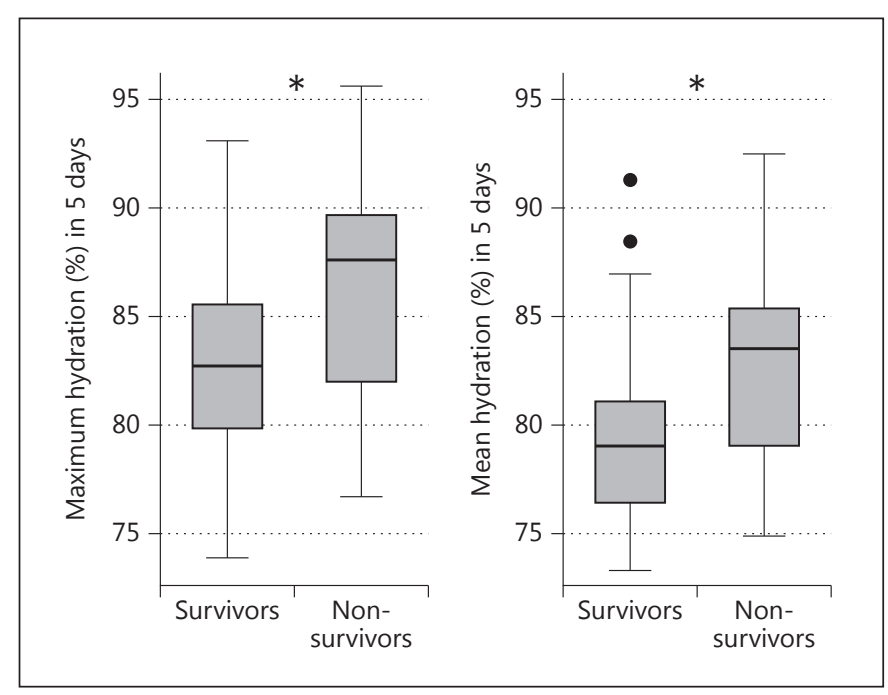

Fig. 3. Box plot of mean and maximum hydration reached during the 5 days in the ICU according to survival outcome. ${ }^{*} \mathrm{p}<0.05$.

pared conservative and liberal fluid management in 1,000 patients with acute lung injury in the ICU. They reported that conservative treatment improved lung function and decreased the duration of mechanical ventilation.

The SOAPS (Sepsis Occurrence in Acutely Ill Patients) study, an important multicenter observational trial, showed that the balance of fluids together with SAPS and age are independent predictors of mortality $[1,23]$. There also appears to be a significant relationship between fluid

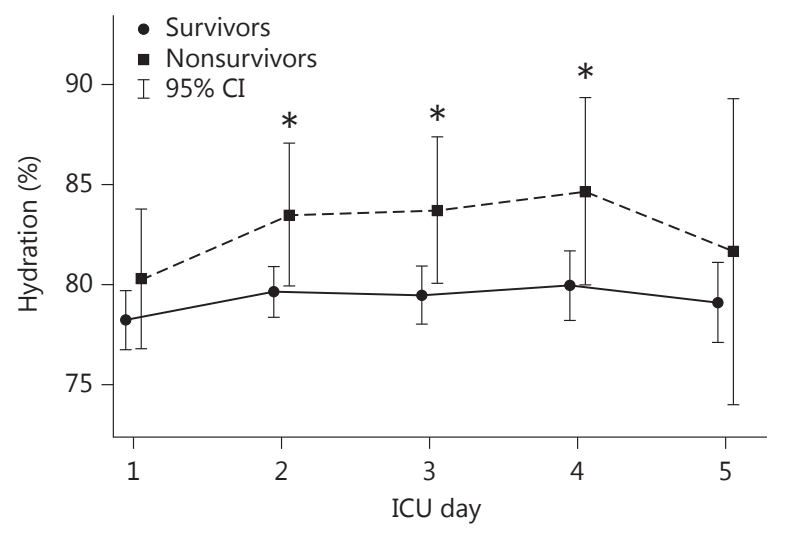

Fig. 4. Mean hydration status during the 5-day observation period for survivors and nonsurvivors in the ICU $(* p<0.05$, Mann-Whitney U test).

balance and sepsis $[1,6]$. Given the aforementioned evidence, it seems that an excessive accumulation of fluid and a subsequent state of overhydration - is a predictor of a negative outcome in critically ill patients. Based on these findings, the detection of overhydration and the definition of an ideal dry weight gain are of considerable importance.

A prospective observational study indicated that fluid balance values recorded by ICU staff may not be consistent with actual body weight measurements. It is therefore suggested that clinical decisions should be based on other techniques that reflect changes in total body water [6]. In addition, beds in a department of intensive care usually lack the equipment enabling continuous monitoring of the patient's weight.

In this context, BIVA can allow for direct assessment of an ICU patient's hydration status and, as an instantaneous measurement, it can bypass possible errors in the recording of fluid balance. A further advantage of serial BIVA measurements is the possibility to establish a target of ideal weight that allows to guide the management of diuretic therapy or ultrafiltration in CRRT patients. This could be helpful in ensuring an adequate circulating blood volume and preventing complications during fluid removal [30].

We performed a prospective assessment of hydration status using BIVA measurements in adult patients admitted to the ICU. A first objective was to assess the degree of hydration in critically ill patients on the 1 st day of ICU hospitalization and examine the evolution of this param- 
eter during hospitalization. A second aim was to investigate how hydration influenced ICU and 60-day mortality.

The main findings from our study are:

- On the 1st day in the ICU, patients showed a marked tendency towards overhydration. Hyperhydration occurred in $>70 \%$ of the patients. This tendency was more marked in patients on CRRT.

- Hyperhydration persisted during the ICU stay. In addition, patients on CRRT showed a significantly worse state of hyperhydration compared to the other patients ( $\mathrm{p}<0.05)$ from the 2nd day of hospitalization onwards. Patients who did not survive in the ICU had significantly higher daily mean hydration on days $2-4$ $(p<0.05)$. The difference on the 5th day was not significant, but this is probably due to the smaller sample size given that many patients were not included in the analysis on day 5 (either because of death or discharge).

- Nonsurvivors showed worse hyperhydration patterns in comparison to survivors ( $\mathrm{p}<0.05$ ). This relationship between hydration and mortality is still present after controlling for the effect of a worse prognosis approximated by any of the three ICU scoring systems (APACHE II, SAPS II or SOFA).

- Mean and maximum hydration levels present a stronger correlation with mortality than with mean and maximum cumulative fluid balance reached during the observation period. Also, in this case, the relationship was confirmed by stratifying patients for the severity of their injury.

The mortality rate in the study population was very high, reflecting the severity of the patients' conditions on admission, as documented by the high values of ICU scoring systems (table 1). Overall mortality was $25 \%$ and was much higher (42.9\%) in patients who required CRRT.

One of the key points of our study is to provide evidence that the accumulation of liquids (measured by BIVA) is particularly common in patients admitted to the ICU and persists throughout the period of observation (fig. 1). This is even more evident in patients who require CRRT, although this technique enables to remove fluid mechanically by ultrafiltration. This result can be explained by two dynamics: (i) the absence of a dry weight target to guide ultrafiltration management in the critically ill patient, and (ii) the hemodynamic instability in patients who undergo CRRT, which does not allow appropriate fluid removal.

In fact, the progressive increase in hydration during the first days of ICU hospitalization probably reflects the common clinical practice of using copious infusions from the moment of first contact between the patient and units of first aid and in the early days of ICU admission. Such management strategy has the purpose of supporting circulation, in order to ensure adequate perfusion to organs and tissues, and preserve normal diuresis. It is known that virtually all patients receive the infusion of a variable amount of fluid during a critical episode [30]. Indeed, there is consensus about the usefulness of administering fluids as soon as possible with the aim to establish specific objectives of cardiovascular stability [24].

At the same time, the literature has already shown that the accumulation of liquids during hospitalization is proportional to the rate of mortality [2]. In particular, there is an increased risk of mortality in CRRT patients who fail to achieve an effective removal of liquid within the first 3 days of treatment [28]. However, the appropriate level of hydration to be achieved in the patient remains to be defined. This could derive from inadequate methods for assessing the state of hydration and may result in a lack of awareness about the state of overhydration in the majority of ICU patients. Ultimately, this practice might lead to untimely intervention in correcting states of severe overhydration.

The findings in our data confirm and expand the evidence in the literature. They support the conclusion that ICU mortality and 60-day mortality show a strong correlation with the achievement of particularly high levels of overhydration. This correlation seems more significant than the one between mortality and cumulative fluid balance, even considering our relatively small sample size. This result points towards the importance of overhydration as a prognostic factor. Thus, hydration status should be carefully considered as an additional parameter in the clinical management of the critically ill patient.

BIVA utilization in the ICU setting is also subject to some limitations. First of all, it is difficult to determine if water overload has a precise pathogenesis in increasing mortality or is merely a marker of disease. Furthermore, patients in the most severe conditions are usually subject to a decrease in peripheral resistance that leads to an overload of fluid in the interstitium; this is measured by BIVA as a state of overhydration, even if there is a state of relative hypovolemia at the central level. As such, the hyperhydration level of the patient may end up being overestimated.

\section{Conclusions}

Our study demonstrates that fluid overload is a frequent condition in critically ill patients. This condition of hyperhydration often persists during ICU hospitaliza- 
tion, independently from the need of administering CRRT. Furthermore, both the average and the maximum hydration reached during the observation period represent a significant risk factor for mortality, both in the ICU and in a 60-day horizon. These findings suggest the need for increased attention to this clinical issue, and the necessity to develop adequate protocols for monitoring the hydration state in ICU patients. Based on the current clinical-structural methods, measurement of the hydration status through BIVA can constitute an additional methodology to achieve correct monitoring of a patient's fluid balance. It may also provide insight into the patient's ideal weight, hence driving a more appropriate therapy man- agement by ultrafiltration and diuretics in critically ill patients. This can ensure an adequate circulating blood volume and prevent complications during fluid removal.

\section{Acknowledgment}

We thank Ulrike Kotanko, MD, for her editorial help.

\section{Disclosure Statement}

No authors have reported any conflict of interest.

\section{References}

1 Payen D, de Pont AC, Sakr Y, Spies C, Reinhart K, Vincent JL: A positive fluid balance is associated with a worse outcome in patients with acute renal failure. Crit Care 2008;12: R74.

-2 Bouchard J, Soroko SB, Chertow GM, Himmelfarb J, Ikizler TA, Paganini EP, Mehta RL: Fluid accumulation, survival and recovery of kidney function in critically ill patients with acute kidney injury. Kidney Int 2009;76:422-427.

3 Wiedemann HP, Wheeler AP, Bernard GR, Thompson BT, Hayden D, deBoisblanc B, Connors AF Jr, Hite RD, Harabin AL: Comparison of two fluid-management strategies in acute lung injury. N Engl J Med 2006;354: 2564-2575.

4 Grams ME, Estrella MM, Coresh J, Brower RG, Liu KD: Fluid balance, diuretic use, and mortality in acute kidney injury. Clin J Am Soc Nephrol 2011;6:966-973.

5 Mehta RL, Clark WC, Schetz M: Techniques for assessing and achieving fluid balance in acute renal failure. Curr Opin Crit Care 2002; 8:535-543.

6 Perren A, Markmann M, Merlani G, Marone C, Merlani P: Fluid balance in critically ill patients. Should we really rely on it? Minerva Anestesiol 2011;77:802-811.

7 McGee S, Abernethy WB 3rd, Simel DL: The rational clinical examination. Is this patient hypovolemic? JAMA 1999;281:1022-1029.

8 Palazzo M: Circulating volume and clinical assessment of the circulation. Br J Anaesth 2001;86:743-746.

$\checkmark 9$ Don C, Burns KD, Levine DZ: Body fluid volume status in hemodialysis patients: the value of the chest radiograph. Can Assoc Radiol J 1990;41:123-126.

10 Kushner RF, Schoeller DA, Fjeld CR, Danford $\mathrm{L}$ : Is the impedance index (ht2/R) significant in predicting total body water? Am J Clin Nutr 1992;56:835-839.

11 Piccoli A, Nigrelli S, Caberlotto A, Bottazzo S, Rossi B, Pillon L, Maggiore Q: Bivariate normal values of the bioelectrical impedance vector in adult and elderly populations. Am J Clin Nutr 1995;61:269-270.
12 Piccoli A: Identification of operational clues to dry weight prescription in hemodialysis using bioimpedance vector analysis. The Italian Hemodialysis-Bioelectrical Impedance Analysis (HD-BIA) Study Group. Kidney Int 1998; 53:1036-1043.

13 Piccoli A: Bioelectric impedance vector distribution in peritoneal dialysis patients with different hydration status. Kidney Int 2004;65: 1050-1063.

4 Pillon L, Piccoli A, Lowrie EG, Lazarus JM, Chertow GM: Vector length as a proxy for the adequacy of ultrafiltration in hemodialysis. Kidney Int 2004;66:1266-1271.

15 Piccoli A: Bioelectric impedance measurement for fluid status assessment. Contrib Nephrol 2010;164:143-152.

16 Nescolarde L, Piccoli A, Roman A, Nunez A, Morales R, Tamayo J, Donate T, Rosell J: Bioelectrical impedance vector analysis in haemodialysis patients: relation between oedema and mortality. Physiol Meas 2004;25:1271-1280.

17 Roos AN, Westendorp RG, Brand R, Souverijn $\mathrm{JH}$, Frolich M, Meinders AE: Predictive value of tetrapolar body impedance measurements for hydration status in critically ill patients. Intensive Care Med 1995;21:125-131.

18 Foley K, Keegan M, Campbell I, Murby B, Hancox D, Pollard B: Use of single-frequency bioimpedance at $50 \mathrm{kHz}$ to estimate total body water in patients with multiple organ failure and fluid overload. Crit Care Med 1999;27:1472-1477.

19 Bellomo R, Ronco C, Kellum JA, Mehta RL, Palevsky P: Acute renal failure - definition, outcome measures, animal models, fluid therapy and information technology needs: the Second International Consensus Conference of the Acute Dialysis Quality Initiative (ADQI) Group. Crit Care 2004;8:R204-R212.

20 Valle R, Aspromonte N, Milani L, Peacock FW, Maisel AS, Santini M, Ronco C: Optimizing fluid management in patients with acute decompensated heart failure (ADHF): the emerging role of combined measurement of body hydration status and brain natriuretic peptide (BNP) levels. Heart Fail Rev 2011;16:519-529.
21 Piccoli A, Rossi B, Pillon L, Bucciante G: A new method for monitoring body fluid variation by bioimpedance analysis: the RXc graph. Kidney Int 1994;46:534-539.

22 Sergi G, Bussolotto M, Perini P, Calliari I, Giantin V, Ceccon A, Scanferla F, Bressan M, Moschini G, Enzi G: Accuracy of bioelectrical impedance analysis in estimation of extracellular space in healthy subjects and in fluid retention states. Ann Nutr Metab 1994;38:158165 .

23 Vincent JL, Sakr Y, Sprung CL, Ranieri VM, Reinhart K, Gerlach H, Moreno R, Carlet J, Le Gall JR, Payen D: Sepsis in European intensive care units: results of the SOAP study. Crit Care Med 2006;34:344-353.

24 Boyd JH, Forbes J, Nakada TA, Walley KR, Russell JA: Fluid resuscitation in septic shock: a positive fluid balance and elevated central venous pressure are associated with increased mortality. Crit Care Med 2011:39:259-265.

25 Schuller D, Mitchell JP, Calandrino FS, Schuster DP: Fluid balance during pulmonary edema. Is fluid gain a marker or a cause of poor outcome? Chest 1991;100:1068-1075.

-26 Upadya A, Tilluckdharry L, Muralidharan V, Amoateng-Adjepong Y, Manthous CA: Fluid balance and weaning outcomes. Intensive Care Med 2005;31:1643-1647.

27 Epstein CD, Peerless JR: Weaning readiness and fluid balance in older critically ill surgical patients. Am J Crit Care 2006;15:54-64.

28 Alsous F, Khamiees M, DeGirolamo A, Amoateng-Adjepong Y, Manthous CA: Negative fluid balance predicts survival in patients with septic shock: a retrospective pilot study. Chest 2000;117:1749-1754.

29 Sakr Y, Vincent JL, Reinhart K, Groeneveld J, Michalopoulos A, Sprung CL, Artigas A, Ranieri VM: High tidal volume and positive fluid balance are associated with worse outcome in acute lung injury. Chest 2005;128:3098-3108.

30 Bagshaw SM, Brophy PD, Cruz D, Ronco C: Fluid balance as a biomarker: impact of fluid overload on outcome in critically ill patients with acute kidney injury. Crit Care 2008;12: 169 